\title{
Box-Particle Implementation and Comparison of Cardinalized Probability Hypothesis Density Filter
}

\author{
Li-ping SONG, Meng LIANG, Hong-bing JI \\ Dept. of Electronic Engineering, Xidian University, No. 2, South Taibai Road, Xi'an, China \\ lpsong@xidian.edu.cn, mliang@stu.xidian.edu.cn, hbji@xidian.edu.cn
}

Manuscript received July 21, 2015

\begin{abstract}
This paper develops a box-particle implementation of cardinalized probability hypothesis density filter to track multiple targets and estimate the unknown number of targets. A box particle is a random sample that occupies a small and controllable rectangular region of nonzero volume in the target state space. In box-particle filter the huge number of traditional point observations is instead by a remarkably reduced number of interval measurements. It decreases the number of particles significantly and reduces the runtime considerably. The proposed algorithm based on box-particle is able to reach a similar accuracy to a Sequential Monte Carlo cardinalized probability hypothesis density (SMC-CPHD) filter with much less computational costs. Not only does it propagates the PHD, but also propagates the cardinality distribution of target number. Therefore, it generates more accurate and stable instantaneous estimates of target number as well as target state than the box-particle probability hypothesis density (BP-PHD) filter does especially in dense clutter environment. Comparison and analysis based on the simulations in different probability of detection and different clutter rate have been done. The effectiveness and reliability of the proposed algorithm are verified by the simulation results.
\end{abstract}

\section{Keywords}

Multi-target tracking, CPHD filter, PHD filter, boxparticle filter, interval measurements

\section{Introduction}

Multi-target tracking has drawn much attention for its significant role in military and civilian fields. In a multiple target environment, the target number as well as the target states is important unknown information. How to track multiple targets with varying number during a clutter environment has been a difficult research issue in both academic and engineering fields for a long time. With finite set statistics (FISST), a theoretic approach in which targets and measurements are modeled by random finite sets (RFS) [1] was introduced by Mahler recently. This approach allows multi-target tracking in the presence of clut- ter and with uncertain associations to be cast in a Bayes filter. Based on this theory, Mahler then proposed the probability hypothesis density (PHD) filter [2] and the cardinalized probability hypothesis density (CPHD) filter [3]. Compared with the PHD filter, the CPHD filter relaxes the Poisson assumptions in target and measurement number to achieve better estimation performance. Plenty of works have been done for their implementations [4-7], such as the Sequential Monte Carlo (SMC) approximation and the Gaussian mixture (GM) approximation. In order to achieve a satisfactory performance a large number of weighted particles are needed to approximate the intensity function in the SMC implementation. It results in high computational complexity and more execution time.

In recent years, many practical applications such as the wireless sensor networks quantized their measurements to only a few bits to reduce the communication bandwidth. Obviously, the standard measurement model is not adequate in this case. In order to solve these problems, the concept of box-particle (BP) filter [8], [9] based on the practical application background is proposed by Amadou Gning, Branko Ristic, and Lyudmila Mihaylova recently. In this approach the huge number of traditional point observations was substituted by a remarkably reduced number of interval measurements. It would certainly reduce the runtime without a lot loss in the performance. An interval measurement expresses a type of uncertainty which is referred as the set-theoretic uncertainty, vagueness or imprecision. The box-particle filter was studied and explained through the Bayesian perspective by interpreting each box particle as a uniform probability density function (PDF) [10]. A single target box-particle Bernoulli filter with box measurements was presented in [11]. The box-particle PHD filter for multi-target tracking with an unknown number of targets, clutter and false alarms was derived in [12].The box-particle cardinality balanced multi-target multi-Bernoulli filter and its implementation was proposed in [13]. The crowd target tracking based on box-particle filter is proposed in [19]. Reference [21] presents an implementation of box-particle filter on extended target. Various works have shown that the box-particle filter can reach a similar performance as the traditional particle filter with less computational complexity and runtime [11-13], [18]. 
The main contribution of this paper is that a new kind of implementation of the cardinalized probability hypothesis density (CPHD) filter, the box-particle cardinalized probability hypothesis density (BP-CPHD) filter is proposed. The approach which is suitable to deal with interval measurements can track multiple targets and estimate the unknown number of targets with low computational complexity and good performance. A comparison of the boxparticle probability hypothesis density (BP-PHD) filter, the BP-CPHD filter and the SMC-CPHD filter is performed. Both the two box-particle methods share a remarkably decreased number and less runtime than the SMC-CPHD filter. The BP-CPHD filter has a more accurate and stable instantaneous estimates of the target number than the BP-PHD filter, especially when the probability of the detection decreases and more clutter appears. Thus, it leads to smaller OSPA distance.

The rest of the paper is organized as follows. The necessary backgrounds on interval methodology, the RFS theory and the CPHD filter are described in Sec. 2. The box-particle PHD filter and the box-particle CPHD filter are presented in Sec. 3. Simulation results are performed in Sec. 4. Finally, conclusions are drawn in Sec. 5.

\section{Background}

This section introduces the random finite set, interval analysis and the SMC-CPHD filter.

\subsection{Random Finite Sets}

In a multiple target scenario, the number of the target may vary over time due to the appearance or disappearance of a target. As a result, the dimensions of the state space vary with the number of targets. Since the number of targets and the number of measurements are a random process, the state set and the observation set can be represented by the RFSs of multi-target state space and multitarget observation space, respectively,

$$
\begin{aligned}
& \mathrm{Z}_{k}=\left\{\mathrm{x}_{k}^{1}, \ldots, \mathrm{x}_{k}^{N(k)}\right\} \in F(\mathrm{X}), \\
& \mathrm{Z}_{k}=\left\{\mathrm{z}_{k}^{1}, \ldots, \mathrm{z}_{k}^{M(k)}\right\} \in F(\mathrm{Z}),
\end{aligned}
$$

where $N(k)$ and $M(k)$ is the number of targets and the number of measurements at time $k . F(\mathrm{X})$ and $F(\mathrm{Z})$ denote the sets of state space $X$ and the observation space $Z$, respectively. In this paper, we assume that if targets or clutter are detected, the sensor does not report the conventional measurement $Z_{k}$ and it will report a closed interval $\left[Z_{k}\right]$ instead, which contains the target originated point measurement $Z_{k}$ with some probability. The set of all such closed intervals on $\mathrm{Z}$, denoted by IZ is the interval measurement space. Due to the imperfect detection process $M(k) \geq 0$ interval measurement $[z]_{k}^{1}, \ldots,[z]_{k}^{M(k)}$ are collected at time $k$. The interval measurements can be represented by a finite set:

$$
\Upsilon_{k}=\left\{[z]_{k}^{1}, \ldots,[z]_{k}^{M(k)}\right\} \in F(\mathrm{IZ})
$$

where $F(I Z)$ is the space of finite subsets of IZ. If the state RFS at time $k-1$ is $\mathrm{X}_{k-1}$, and the state RFS $\mathrm{X}_{k}$ at time $k$ can be expressed by

$$
\mathbf{X}_{k}=\left(\bigcup_{\mathbf{x} \in \mathbf{X}_{k-1}} S_{k \mid k-1}(\mathbf{x})\right) \bigcup \Gamma_{k}
$$

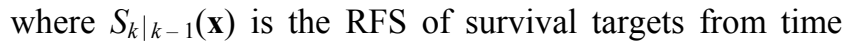
$k-1$ to time $k$, and $\Gamma_{k}$ is the RFS of targets which birth at time $k$. The target spawned by a previous target is not considered in this paper. The multi-target measurements $\Upsilon_{k}$ is modeled by RFS as

$$
\Upsilon_{k}=\kappa_{k} \bigcup\left(\bigcup_{\mathbf{x} \in \mathbf{X}_{k}} \Theta_{k}(\mathbf{x})\right)
$$

where $\Theta_{k}(\mathbf{x})$ is the measurement set generated by the true targets, and $\kappa_{k}$ represents the measurement set from the clutter. To deal with the heavy computational burden in processing the joint probability density function of $\mathrm{X}_{k}$ and $\mathrm{Z}_{k}$ directly, Mahler proposed the probability hypothesis density (PHD) filter which can approximate the probability density of multi-target RFS with its first-order moment [2]. Thereafter, he proposed the cardinalized probability hypothesis density (CPHD) filter [3]. These two filters can be implemented by GM or SMC approximations.

\subsection{Interval Analysis}

This section will briefly introduce the interval analysis which used in this paper. More details on this field are available in [14]. The original idea of interval analysis is to deal with intervals of real instead of real numbers for exact computation in the presence of rounding errors. Box-particle filter based on interval analysis is suitable to deal with the interval measurements. A real interval $[x]=[x, \bar{x}]$ is defined as a closed and connected subset of the set of real numbers. $\underline{x}$ and $\bar{x}$ represent the lower bound and the upper bound of the interval. The arithmetic operations between numbers and the operations between sets have been extended to intervals. In a multi-dimensional vector form, it becomes a box $[x]$ defined as a Cartesian product of $l$ intervals: $[x]=\left[x_{1}\right] \times\left[x_{2}\right] \ldots \times\left[x_{l}\right]$. In this paper, the operator $|[\cdot]|$ will be used as the volume $|[x]|$ of a box $[x]$. And mid $([x])$ denotes the center of a box.

A box $[x]$ through a nonlinear transformation in general has a non-box shape. In order to remain in the realm of boxes, an inclusion function of a given function $f$ is defined such that the image of a box $[x]$ is a box $[f]([x])$ containing $f([x])$. The inclusion function is used so that the size of the box $[f]([x])$ is minimal but still covers the whole image of the box $[x]$ in this paper. The inclusion function is able to reduce the calculation and make the process to converge faster. Another significant concept is contraction [8], which 
will be used in the definition of likelihood functions and the update step of the proposed filters. In this paper, the Constraint Propagation (CP) [13] will be used, for the sake of its good suitability in the context of tracking problems.

\subsection{The SMC-CPHD Filter}

The cardinalized probability hypothesis density (CPHD) filter which relaxes the Poisson assumptions in target and measurement number is proposed by Mahler. Not only does it propagate the PHD, but also propagates the cardinality distribution of target number. Therefore, it generates more accurate and stable instantaneous estimates of the target number and admits more false alarm processes than the PHD filter does. Both the Sequential Monte Carlo (SMC) and the Gaussian mixture (GM) approximations can be used to implement the CPHD filter. In this section we will introduce the SMC approximation. The description and detailed analysis of SMC-CPHD is available in [7]. The evaluation for SMC-CPHD has been done using ground truth data obtained by a marker-based motion capture system [23]. The SMC-CPHD recursion is briefly summarized as follows:

1) SMC-CPHD prediction: The distribution of the target number as well as the target state must also be propagated. Assume that the particle set from the previous time $k-1$ is $\left\{\left(w_{k-1}^{p e r,(i)}, x_{k-1}^{p e r,(i)}\right)\right\}_{i=1}^{N_{k-1}}$, where $w_{k-1}^{\text {per, (i) }}$ is the corresponding weights of the persistent particles. $N_{k-1}$ represents the number of particles. The newborn particle set is $\left\{w_{k-1}^{b i r,(m)}, \mathbf{x}_{k-1}^{b i r,(m)}\right\}_{m=1}^{N_{k, \text { new }}} \cdot w_{k-1}^{\text {bir, }(m)}$ and $N_{k, \text { new }}$ represents the corresponding weights and number of newborn particles.

$$
\begin{gathered}
\left\{w_{k-1}^{(i)}, \mathbf{x}_{k-1}^{(i)}\right\}_{i=1}^{N_{k}}= \\
\left\{w_{k-1}^{\text {per },(n)}, \mathbf{x}_{k-1}^{\text {per, }(n)}\right\}_{n=1}^{N_{k-1}} \bigcup\left\{w_{k-1}^{b i r,(m)}, \mathbf{x}_{k-1}^{b i r,(m)}\right\}_{m=1}^{N_{k, n e w}} \\
\mathbf{x}_{k \mid k-1}^{(i)}=f_{k \mid k-1}\left(\cdot \mid \mathbf{x}_{k-1}^{(i)}\right), i=1, \cdots, N_{k}, \\
w_{k \mid k-1}^{(i)}=\mathrm{P}_{\mathrm{S}}\left(\mathbf{x}_{k-1}^{(i)}\right) w_{k-1}^{(i)}, i=1, \cdots, N_{k}, \\
p_{k / k-1}(n)=\sum_{n^{\prime}=0}^{\infty} p_{k-1}\left(n^{\prime}\right) M\left(n, n^{\prime}\right), \\
M\left(n, n^{\prime}\right)=\sum_{i=0}^{\min \left\{n, n^{\prime}\right\}} p_{b i r t h}(n-i)\left(\begin{array}{l}
n^{\prime} \\
i
\end{array}\right) d^{m^{\prime}-i}(1-d)^{i} .
\end{gathered}
$$

$N_{k}=N_{k-1}+N_{k \text {, new }}$ is the whole number of the particles. $f_{k \mid k-1}\left(\cdot \mid \mathbf{x}_{k-1}^{(i)}\right)$ and $\mathrm{P}_{\mathrm{S}}\left(\mathbf{x}_{k-1}^{(i)}\right)$ are the single target transition density and the probability of target survival, respectively. $p_{k \mid k-1}(n)$ is the predicted cardinality distribution of the target number. $M$ is the transfer matrix and $p_{\text {birth }}(n)$ is the probability for $n$ new targets to appear between scan $k-1$ and $k$ derived from the birth model.

2) SMC-CPHD update: Assume that the predicted particle set at time $k$ is $\left\{w_{k / k-1}^{(i)}, \mathbf{x}_{k / k-1}^{(i)}\right\}_{i=1}^{N_{k}}$. The update equation of the state intensity and the cardinality distribution is realized as follows. $p_{c}(m)$ denotes the probability for $m$ false alarms.

$$
\begin{gathered}
\hat{w}_{\mathrm{k}}^{(i)}=\left[\left(1-\mathrm{P}_{D}\right) \frac{\mathrm{L}\left(\mathrm{Z}_{k} \mid-\mathrm{D}\right)}{\mathrm{L}\left(\mathrm{Z}_{k}\right)}+\mathrm{P}_{D} \frac{\mathrm{L}\left(\mathrm{Z}_{k} \mid \mathrm{D}\right)}{\mathrm{L}\left(\mathrm{Z}_{k}\right)}\right] w_{k \mid k-1}^{(i)}, \\
p_{k}(n)=\frac{\mathrm{L}\left(\mathrm{Z}_{k} \mid n\right)}{\mathrm{L}\left(\mathrm{Z}_{k}\right)} p_{k / k-1}(n)
\end{gathered}
$$

where the conditions $\mathrm{D}$ and $-\mathrm{D}$ are short hand notations for target detected and not detected, respectively. L[·] denotes likelihood function. The likelihood ratios above can be found in [15]. In this paper, the interval measurement is used in the SMC-CPHD filter. Thus the generalized likelihood is different from the traditional SMC-CPHD filter. The generalized likelihood function under uncertain measurement satisfies the following formula [21]. Assume that $\inf ([z])$ and $\sup ([z])$ represent the lower and upper limits of the interval measurement $[z]$, respectively.

$$
\phi(x, \mu, Q)=\int_{-\infty}^{\mathrm{x}} N(t, \mu, Q) d t
$$

$\phi(\mathrm{x}, \mu, Q)$ represents the cumulative distribution function of Gaussian distribution $N(\mathrm{t}, \mu, Q)$ with mean $\mu$ and variance $Q$. Therefore, the generalized likelihood of the SMC-CPHD filter under interval measurement is as follows. $R$ is the variance of Gaussian distribution $N\left(\mathrm{z}, x_{k \mid k-1}, Q\right)$.

$$
\begin{aligned}
& \mathrm{L}_{k}\left([\mathbf{z}] \mid \mathbf{x}_{k \mid k-1}\right)=\int_{[\mathrm{z}]} N\left(\mathrm{z}, \mathbf{x}_{k \mid k-1}, \mathrm{R}\right) d \mathrm{z} \\
& =\int_{\inf [\mathrm{z}]}^{\sup [\mathrm{z}]} N\left(\mathrm{z}, \mathbf{x}_{k \mid k-1}, \mathrm{R}\right) d z \\
& =\phi\left(\sup [\mathrm{z}], \mathrm{x}_{k \mid k-1}, \mathrm{R}\right)-\phi\left(\inf [\mathrm{z}], \mathrm{x}_{k \mid k-1}, \mathrm{R}\right)
\end{aligned}
$$

Applying this to equation (11), we can easily get the weight of the particle. The generalized likelihood of the BP-CPHD filter is different from that of the SMC-CPHD filter. It will be introduced in the following of the paper.

\section{Implementations}

For varying number multi-target tracking in clutter environment, PHD and CPHD filter based on random finite sets provides good solutions [2], [3]. Their implementations include Sequential Monte Carlo (SMC) approximation and the Gaussian mixture (GM) approximation. Boxparticle filter emerging recently provides a new implementation for PHD filter [12], [18]. On the other hand, compared with the PHD filter, the CPHD filter relaxes the Poisson assumptions in target and measurement number to achieve better estimation performance. Therefore, we propose a box-particle implementation for CPHD filter in this section.

\subsection{The Box-Particle PHD Filter}

The sequential Monte Carlo implementation details 
using a box particle representation are presented in the following.

1) Prediction: Suppose that the particle set from the previous time is denoted by $\left\{\left(w_{k-1}^{p e r,(i)},[x]_{k-1}^{p e r,(i)}\right)\right\}_{i=1}^{N_{k-1}}$. $w_{k-1}^{p e r,(i)}$ is the corresponding weight and $N_{k-1}$ represents the number of particles. The newborn particle set $\left\{w_{k-1}^{\text {bir, }(m)},\left[\mathbf{x}_{k-1}^{\text {bir, }(m)}\right]\right\}_{m=1}^{N_{k, n w}}$ is obtained from the measurement $\left[Z_{k-1}\right]$ of the previous scan $k-1$. The number of the newborn particles is $N_{k, \text { new }}$. More details about producing the new particles can be found in [12]. $\left\{w_{k-1}^{(i)},\left[\mathbf{x}_{k-1}^{(i)}\right]\right\}_{i=1}^{N_{k}}=\left\{w_{k-1}^{\text {per, },(n)},\left[\mathbf{x}_{k-1}^{\text {per, },(n)}\right]\right\}_{n=1}^{N_{k-1}} \bigcup\left\{w_{k-1}^{\text {bir, }(m)},\left[\mathbf{x}_{k-1}^{\text {bir, }(m)}\right]\right\}_{m=1}^{N_{k, n e w}}$ $N_{k}=N_{k-1}+N_{k, \text { new }}$ is the whole number of the box-particles. These particles mentioned above are propagated through the motion model and inclusion function. The survival probability is $P_{S}$.

$$
\begin{gathered}
{\left[\mathbf{x}_{k \mid k-1}^{(i)}\right]=\left[f_{k \mid k-1}\right]\left(\cdot \mid\left[\mathbf{x}_{k-1}^{(i)}\right]\right), i=1, \cdots, N_{k},} \\
w_{k \mid k-1}^{(i)}=\mathrm{P}_{\mathrm{S}}\left(\left[\mathbf{x}_{k-1}^{(i)}\right]\right) w_{k-1}^{(i)}, i=1, \cdots, N_{k} .
\end{gathered}
$$

2) Update: The update of the state intensity is realized as follows, which has a similar scheme of the SMC-PHD filter. The probability of target detection $\mathrm{P}_{D}$ is assumed to be constant, and the number of false detections per scan is modeled by a Poisson distribution with mean $\lambda$. The prior probability of false detection is modeled by $c\left(\left[\mathbf{z}_{j}\right]\right)$.

$$
\hat{w}_{\mathrm{k}}^{(i)}=\left[\begin{array}{l}
\left(1-\mathrm{P}_{\mathrm{D}}\left(\left[\mathbf{x}_{k \mid k-1}^{(i)}\right]\right)\right) \\
+\sum_{j=1}^{m_{k}} \frac{g_{k}\left(\left[\mathbf{z}_{j}\right] \mid\left[\mathbf{x}_{k \mid k-1}^{(i)}\right]\right) \mathrm{P}_{\mathrm{D}}\left(\left[\mathbf{x}_{k \mid k-1}^{(i)}\right]\right)}{\lambda_{k / k-1}\left(\left[\mathbf{z}_{j}\right]\right)}
\end{array}\right] \cdot w_{k \mid k-1}^{(i)} \cdot
$$

A different likelihood function from that of SMCPHD filter is presented here.

$$
g_{k}\left(\left[\mathbf{z}_{j}\right] \mid\left[\mathbf{x}_{k \mid k-1}^{(i)}\right]\right)=\frac{\left|\left[h_{c p}\right]\left(\left[\mathbf{x}_{k \mid k-1}^{(i)}\right],\left[\mathbf{z}_{j}\right]\right)\right|}{|[\mathbf{x}]|} .
$$

The function $\left[h_{c p}\right]\left(\left[\mathbf{x}_{k \mid k-1}^{(i)}\right],\left[\mathbf{z}_{j}\right]\right)$ returns a contracted version of $\left[\mathbf{x}_{k \mid k-1}^{(i)}\right]$ with its corresponding measurement $\left[\mathbf{z}_{j}\right]$, if no $\left[\mathbf{z}_{j}\right]$ is found, this particle is not contracted. The contraction approach here we used is: $[y]=[y] \cap\left[y_{z}\right]$ $[x]=[x] \cap\left[x_{z}\right]$. The contraction approach is widely used. Reference [18] provides a contraction example. More information on contraction step can be found in [16]. For interval measurements $\left[\mathbf{z}_{j}\right]$, compute the correction term below:

$$
\lambda_{k / k-1}\left(\left[\mathbf{z}_{j}\right]\right)=\left[\begin{array}{l}
\lambda c\left(\left[\mathbf{z}_{j}\right]\right)+ \\
\sum_{i=1}^{N_{k-1}+N_{k, n e w}} g_{k}\left(\left[\mathbf{z}_{j}\right] \mid\left[\mathbf{x}_{k \mid k-1}^{(i)}\right]\right) \mathrm{P}_{\mathrm{D}}\left(\left[\mathbf{x}_{k \mid k-1}^{(i)}\right]\right) w_{k \mid k-1}^{(i)}
\end{array}\right]
$$

Normalize the weights

$$
\hat{w}_{k}^{(i)}=\frac{\hat{w}_{k}^{(i)}}{\sum_{i=1}^{N_{k}} \hat{w}_{k}^{(i)}} .
$$

3) Estimate: The state and number of the targets is estimated in this step.

$$
\begin{gathered}
\hat{\mathrm{x}}_{\mathrm{k}}=\sum_{i=1}^{N_{k}} w_{k}^{i} \cdot \operatorname{mid}\left(\left[\mathrm{x}_{k}^{i}\right]\right), \\
\hat{\mathrm{P}}_{\mathrm{k}}=\sum_{i=1}^{N_{k}} w_{k}^{i}\left(\left(\mathrm{x}_{k}^{i}-\operatorname{mid}\left(\left[\mathrm{x}_{k}^{i}\right]\right)\right) \cdot\left(\mathrm{x}_{k}^{i}-\operatorname{mid}\left(\left[\mathrm{x}_{k}^{i}\right]\right)\right)^{\mathrm{T}}\right), \\
\hat{N}_{k}=\operatorname{int}\left(\sum_{i=1}^{N_{k}} \hat{w}_{k}^{(i)}\right) .
\end{gathered}
$$

$\operatorname{mid}[\cdot]$ means finding the center of the box $[\cdot] . \hat{N}_{k}$ is the estimated number of the target. In prediction and update steps, the first order moment of multi-target RFS, rather than multi-target multi-Bernoulli density mentioned in reference [13] is propagated.

4) Resampling:Assume that $N$ is the number of the resampled particles, instead of replicating box-particles which have been selected more than once in the resampling step as the traditional particle filter does, we divide them into $N$ equally weighted box-particles and resample them times to obtain particle set $\left\{w_{k}^{(i)}=\frac{1}{N},\left[x_{k}^{(i)}\right]\right\}_{i=1}^{N}$ from $\left\{\hat{w}_{k}^{(i)},\left[\boldsymbol{x}_{k \mid k-1}^{(i)}\right]\right\}_{i=1}^{N_{k}}$. Here a random dimension is picked to be divided for the box-particle which has been selected. The details are introduced in [13].

5) Clustering: The box particle is changed to point particle according to $\operatorname{mid}\left(\left[x_{k}^{i} \cdot\right]\right)$. For these obtained point particles, we use the K-means clustering method [24] to get $\hat{N}_{k}$ cluster centers as the position of the targets.

\subsection{The Box-Particle CPHD Filter}

The box-particle implementation to the CPHD filter is presented in the following. The basic concepts of box-particle approximation of the CPHD filter are essentially the same as that of the PHD filter.The difference is that the distribution of the target number must also be propagated.

1) Prediction: The states and weights of the particles were propagated through the same approach as the BPPHD filter. Suppose that at time $k-1$ the particle set is $\left\{\left(w_{k-1}^{\text {per },(i)},[x]_{k-1}^{\text {per },(i)}\right)\right\}_{i=1}^{N_{k-1}}$. The newborn particle set $\left\{w_{k-1}^{\text {bir },(m)},\left[\mathbf{x}_{k-1}^{\text {bir, }(m)}\right]\right\}_{m=1}^{N_{k, n e w}}$ is generated from the measurement set from the previous scan $k-1$. In this paper, five particles are produced for each measurement $[\mathbf{z}]$. The newborn particle is generated around the previous measurement with a corresponding weight according to (24). $P_{b}$ is the probability of birth. More details can be found in [12]. These particles mentioned above are propagated through 
the motion model and inclusion function. The survival probability is $P_{\mathrm{S}}$.

$$
\begin{gathered}
w_{k-1}^{b i r,(m)}=\frac{P_{b}}{N_{k, \text { new }}}, m=1, \ldots N_{k, \text { new }}, \\
{\left[\mathbf{x}_{k \mid k-1}^{(i)}\right]=\left[f_{k \mid k-1}\right]\left(\cdot \mid\left[\mathbf{x}_{k-1}^{(i)}\right]\right), i=1, \cdots, N_{k},} \\
w_{k \mid k-1}^{(i)}=\mathrm{P}_{\mathrm{S}}\left(\left[\mathbf{x}_{k-1}^{(i)}\right]\right) w_{k-1}^{(i)}, i=1, \cdots, N_{k} .
\end{gathered}
$$

The prediction equation for the cardinality distribution can be written in terms of a transfer matrix $M$ :

$$
\begin{gathered}
p_{k / k-1}(n)=\sum_{n^{\prime}=0}^{\infty} p_{k-1}\left(n^{\prime}\right) M\left(n, n^{\prime}\right), \\
M\left(n, n^{\prime}\right)=\sum_{i=0}^{\min \left\{n, n^{\prime}\right\}} p_{b i r t h}(n-i)\left(\begin{array}{l}
n^{\prime} \\
i
\end{array}\right) d^{m^{\prime}-i}(1-d)^{i} .
\end{gathered}
$$

$P_{\text {birth }}(n)$ is the probability for $n$ new targets to appear between scan $k-1$ and $k$ derived from the birth model. For a constant scan rate, the matrix $M$ is constant and can be calculated in advance.

2) Update: Assume that the predicted particle set at time $k$ is $\left\{w_{k / k-1}^{(i)},\left[\mathbf{x}_{k / k-1}^{(i)}\right]\right\}_{i=1}^{N_{k}}$. The update equation of the state intensity and the cardinality distribution is realized as follows. $p_{c}(m)$ denotes the probability of $m$ false alarms.

$$
\begin{gathered}
\hat{w}_{\mathrm{k}}^{(i)}=\left[\begin{array}{c}
\left(1-\mathrm{P}_{D}\right) \frac{\mathrm{L}\left(\left[\mathrm{Z}_{k}\right] \mid-\mathrm{D}\right)}{\mathrm{L}\left(\left[\mathrm{Z}_{k}\right]\right)} \\
+\mathrm{P}_{D} \frac{\mathrm{L}\left(\left[\mathrm{Z}_{k}\right] \mid \mathrm{D}\right)}{\mathrm{L}\left(\left[\mathrm{Z}_{k}\right]\right)}
\end{array}\right] w_{k \mid k-1}^{(i)}, \\
p_{k}(n)=\frac{\mathrm{L}\left(\left[\mathrm{Z}_{k}\right] \mid n\right)}{\mathrm{L}\left(\left[\mathrm{Z}_{k}\right]\right)} p_{k / k-1}(n)
\end{gathered}
$$

where the conditions $\mathrm{D}$ and $-\mathrm{D}$ are short hand notations for target detected and not detected, respectively. The likelihood ratios above are given by:

$$
\begin{gathered}
\mathrm{L}_{k}\left(\left[\mathbf{z}_{j}\right] \mid\left[\mathbf{x}_{k \mid k-1}^{(i)}\right]\right)=\frac{\left|\left[h_{c p}\right]\left(\left[\mathbf{x}_{k \mid k-1}^{(i)}\right],\left[\mathbf{z}_{j}\right]\right)\right|}{|[\mathbf{x}]|}, \\
\mathrm{L}\left(\left[\mathrm{Z}_{k}\right] \mid \mathrm{D}\right)=\sum_{j=1}^{m_{k}} l\left(z_{k}^{(s)} \mid x\right) \mathrm{L}\left(\left[\mathrm{Z}_{k}\right] \mid a_{k}^{(j)}=s\right), \\
\mathrm{L}\left(\left[\mathrm{Z}_{k}\right] \mid a_{k}^{(j)}=s\right)=\frac{1}{n_{k / k-1}}\left[\sum_{j=1}^{m_{k}} \beta_{k}^{(j)} \alpha_{k}^{(j)} \ldots\right. \\
\left.\mathrm{L}\left(\left[\mathrm{Z}_{k}\right]\right)=\frac{1}{n_{k / k-1}} \sum_{j=0}^{m_{k}} \alpha^{(j)} \beta^{(j)} \sigma_{j}\left(\left\{L_{k}^{(1)}, \ldots, L_{k}^{\left(m_{k}\right)}\right\} /\left\{L_{k}^{(s)}\right\}\right)\right],
\end{gathered}
$$

$$
\begin{aligned}
\mathrm{L}\left(\left[\mathrm{Z}_{k}\right] \mid n\right)=\sum_{j=0}^{\min \{m, n\}} \beta_{k}^{(j)} \frac{n !\left(1-\mathrm{P}_{\mathrm{D}}\right)^{n-j} \sigma\left(\left\{L_{k}^{(1)}, \ldots, L_{k}^{\left(m_{k}\right)}\right\}\right)}{(n-j) !} \\
\alpha^{(j)}=\sum_{(n-j) !}^{n !} p_{k / k-1}(n)\left(1-\mathrm{P}_{\mathrm{D}}\right)^{n-j} \\
\beta_{k}^{(j)}=p_{c}(m-j) \frac{\left(m_{k}-j\right) !}{m_{k} !} \lambda^{-j}
\end{aligned}
$$

Here, not only the first order moment of multi-target RFS, but also the cardinality distribution is propagated in prediction and update steps.

3) Contraction: A contraction algorithm is used to obtain a contracted version of $\left[\mathbf{x}_{k \mid k-1}^{(i)}\right]$ with its corresponding measurement $\left[\mathbf{z}_{j}\right]$. Given the contraction function $\left[h_{c p}\right]\left(\left[\mathbf{x}_{k \mid k-1}^{(i)}\right],\left[\mathbf{z}_{j}\right]\right), \quad$ and $[\mathbf{x}]=[x] \times[\dot{x}] \times[y] \times[\dot{y}] \quad$, $[\mathrm{z}]=\left[x_{z}\right] \times\left[y_{z}\right]$, if no $\left[\mathbf{z}_{j}\right]$ is found, this particle is not contracted. The contraction approach here we used is: $[y]=[y] \cap\left[y_{z}\right],[x]=[x] \cap\left[x_{z}\right]$. More information on contraction step can be found in [16]. Normalized step and the estimates of the target state is the same as that of BP-PHD filter. For the BP-CPHD filter the MAP estimate may produce more accurate and stable estimates of target number $\hat{N}_{k}$. The cardinalized distribution, rather than the weights of particles, is used to estimate the number of targets here. It is different from BP-PHD filter.

$$
\begin{gathered}
\hat{w}_{k}^{(i)}=\frac{\hat{w}_{k}^{(i)}}{\sum_{i=1}^{N_{k}} \hat{w}_{k}^{(i)}}, \\
\hat{N}_{k}=\arg \sup p_{k}(n) .
\end{gathered}
$$

4) Resampling and clustering: Assume that $N$ is the number of the resampled particles, instead of replicating box-particles which have been selected more than once in the resampling step, we divide them into $N$ equally weighted box-particles and resample them times to obtain particle set $\left\{w_{k}^{(i)}=\frac{1}{N},\left[x_{k}^{(i)}\right]\right\}_{i=1}^{N}$ from $\left\{\hat{w}_{k}^{(i)},\left[\boldsymbol{x}_{k \mid k-1}^{(i)}\right]\right\}_{i=1}^{N_{k}}$. In this paper we randomly pick a dimension to be divided for the selected box-particle. The K-means clustering method [24] is still used in the BP-CPHD filter to get the position of the targets. The key steps of the BP-CPHD filter are summarized in Tab. 1.

\section{Numerical Studies}

Numerical studies for the proposed box-particle CPHD filter are given in this section. We evaluate filter performance using the optimum sub pattern assignment (OSPA) distance comparing with the SMC-CPHD filter and BP-PHD filter. Interval measurements are utilized to all the three methods. 
Algorithmic flow of the box-particle CPHD filter

1. Given $\left\{\left(w_{k-1}^{p e r,(i)},[x]_{k-1}^{p e r,(i)}\right)\right\}_{i=1}^{N_{k-1}}$.

2. Create newborn particle set from the previous measurement $\left\{w_{k-1}^{b i r,(m)},\left[\mathbf{x}_{k-1}^{b i r,(m)}\right]\right\}_{m=1}^{N_{k, \text { new }}}$ with corresponding weight defined by (26);

Prediction

3. Propagate the particles $\left[\mathbf{x}_{k \mid k-1}^{(i)}\right]$ for $i=1, \ldots, N_{k}$ according to (27);

4. Compute the weight $w_{k \mid k-1}^{(i)}$ for $i=1, \ldots, N_{k}$ according to (28);

5. Compute the cardinality $p_{k / k-1}(n)$ according to (29);

Update

6. Box particle contraction: $[y]=[y] \cap\left[y_{z}\right],[x]=[x] \cap\left[x_{z}\right]$.

7. Compute the generalized likelihood $\mathrm{L}_{k}\left(\left[\mathbf{z}_{j}\right] \mid\left[\mathbf{x}_{k \mid k-1}^{(i)}\right]\right)$ for every particle $\left\{w_{k / k-1}^{(i)},\left[\mathbf{x}_{k / k-1}^{(i)}\right]\right\}_{i=1}^{N_{k}}$ and every measurement $\left[\mathbf{z}_{j}\right]$ according to (33);

8. Compute the weight $\hat{w}_{k}^{(i)}$ for $i=1, \ldots, N_{k}$ according to (31);

9. Compute the cardinality $p_{k}(n)$ according to (32);

Estimate

10. Get the center of the particle according to $\operatorname{mid}\left(\left[x_{k}^{i}\right]\right)$;

11. Compute the number of the target $\hat{N}_{k}$ according to (41);

12. Use K-means clustering method to get $\hat{N}_{k}$ cluster centers as the position of the targets.

13. Weight normalization: $\hat{w}_{k}^{(i)}$ for $i=1, \ldots, N_{k}$.

14. Resample $N$ equally weighted particles from $\left\{\hat{w}_{k}^{(i)},\left[\boldsymbol{x}_{k \mid k-1}^{(i)}\right]\right\}_{i=1}^{N_{k}}$.

Output

15. Particles obtained from resample $\left\{w_{k}^{(i)}=\frac{1}{N},\left[x_{k}^{(i)}\right]\right\}_{i=1}^{N}$.

Tab. 1. Algorithmic flow of the box-particle CPHD filter.

\subsection{Simulation Setup}

Consider a five target scenario on the surveillance region. The targets are moving according to the nearly constant velocity motion model in two dimensions and the prediction of the persistent particles can be modeled by:

$$
\left[\mathbf{x}_{k+1 \mid k}\right]=\mathbf{F}\left[\mathbf{x}_{k}\right]+\Gamma w .
$$

Here, $\quad \mathbf{F}=\left[\begin{array}{cccc}1 & T & 0 & 0 \\ 0 & 1 & 0 & 0 \\ 0 & 0 & 1 & T \\ 0 & 0 & 0 & 1\end{array}\right], \quad\left[\mathbf{x}_{k}\right]=\left(\begin{array}{c}{[x]} \\ {[\dot{x}]} \\ {[y]} \\ {[\dot{y}]}\end{array}\right), \quad \Gamma=\left[\begin{array}{cc}T^{2} / 2 & 0 \\ T & 0 \\ 0 & T^{2} / 2 \\ 0 & T\end{array}\right]$ $\left[\mathbf{x}_{k}\right]=[[x],[\dot{x}],[y], \dot{y}]^{\mathrm{T}}$ is the target state interval,$([x],[y])$ and $([\dot{x}],[\dot{y}])$ are the target position interval and velocity interval, respectively. The state noise $w$ is white Gaussian noise with a covariance matrix $\boldsymbol{Q}=\operatorname{diag}([0.5,0.1])$. The inclusion functions are hidden in (42) for the individual dimension of the state space. What we can see from (42) is that every variable only appears once for each dimension and all operations are continuous, so these natural inclusion functions are minimal and the propagated boxes have minimal size. The point measurements function is $h_{k}(\mathbf{x})=[x, y]^{T}$, so an interval measurement at time $k$ is defined as:

$$
[\mathbf{z}]_{k}=\left[h_{k}(\mathbf{x})+\mathbf{v}_{k}-0.45 \Delta, \quad h_{k}(\mathbf{x})+\mathbf{v}_{k}+0.55 \Delta\right]
$$

where, assume the interval length is $\boldsymbol{\Delta}=[25,23]^{T}$. The measurement noise $\mathbf{v}_{k}$ is white Gaussian noise with a covariance matrix $\boldsymbol{R}=\operatorname{diag}\left(\left[2.5^{2}, 2.5^{2}\right]\right)$. The initial position, velocity and moving duration of the targets are listed in Tab. 2. In this paper, we needn't to initialize all the targets, for the sake of that the new box particles are generated from the measurements of the previous time. The first target is initialized as follows: $\boldsymbol{m}=[-500,20,-80,-25]^{\mathrm{T}}$, and $\boldsymbol{P}=\operatorname{diag}([10,2,10,2]) . \boldsymbol{m}$ and $\boldsymbol{P}$ represent the mean vector and variance matrix, respectively. For the SMCCPHD filter, 2000 particles are sampled from $N(m, P)$. While the box particle number for the BP-PHD filter and BP-CPHD filter is only 35 . The probability of target survival and target birth is $\mathrm{P}_{\mathrm{S}}=0.99$ and $\mathrm{P}_{b}=0.01$ respectively. The clutter is modeled as a Poisson RFS with the mean $r$ per scan over the surveillance region. The parameters of the OSPA distance are set to be $p=2$ and $c=70$. Figure 1 shows the true target trajectories together with interval measurements in the presence of the clutter in $x-y$ plane. The average computation time of one Monte Carlo trial for the three filters is listed in Tab. 3 .

\begin{tabular}{|c|c|c|c|}
\hline Target & Initial position and velocity & Start time & End time \\
\hline 1 & $(500,20,-80,-25)$ & 1 & 34 \\
\hline 2 & $(-600,18,80,10)$ & 7 & 60 \\
\hline 3 & $(-550,19,-550,10)$ & 25 & 52 \\
\hline 4 & $(200,-20,-40,10)$ & 16 & 40 \\
\hline 5 & $(-300,20,700,-25)$ & 30 & 56 \\
\hline
\end{tabular}

Tab. 2. The initial position, velocity and moving duration.

\begin{tabular}{|c|c|c|c|}
\hline Filter & SMC-CPHD & BP-CPHD & BP-PHD \\
\hline Running time(s) & 166.0843 & 30.0348 & 17.0552 \\
\hline Persistent particle number & 2000 & 35 & 35 \\
\hline Newborn particle number & 500 & 5 & 5 \\
\hline
\end{tabular}

Tab. 3. Average running time and the number of particles.

\subsection{Experiments}

1) Comparing the performance of the three filters with different number of clutter: To evaluate the average performance, 50 Monte Carlo (MC) trials are performed. True targets trajectories and tracking results are shown in Fig. 2. Set the mean number of the clutter $r=3,8,12$, respectively. In this experiment the probability of detection is 


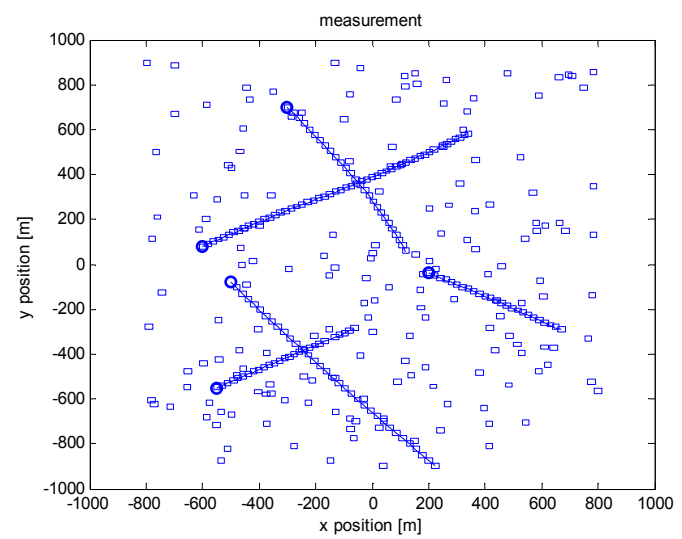

Fig. 1. True target trajectories together with interval measurements in the presence of the clutter in $x-y$ plane. The solid lines are the true target trajectories and the start position for each track is shown with circular. The measurements are visualized as boxes.

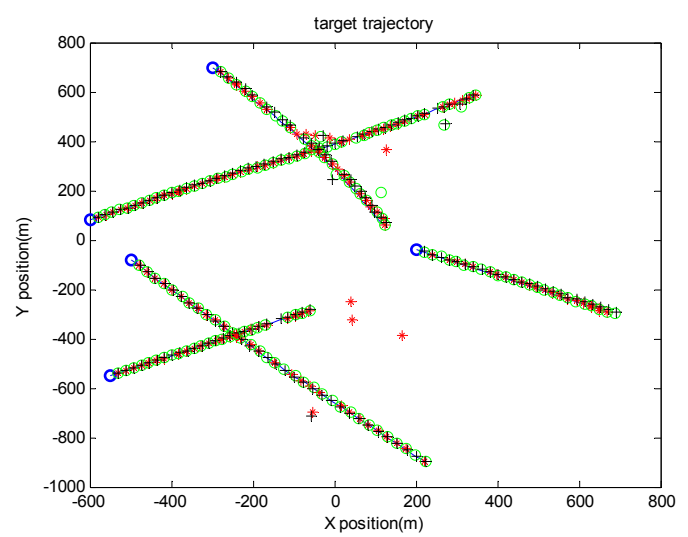

Fig. 2. (a) True targets trajectories and tracking results of SMC-CPHD (+), BP-PHD (*) and BP-CPHD (o).
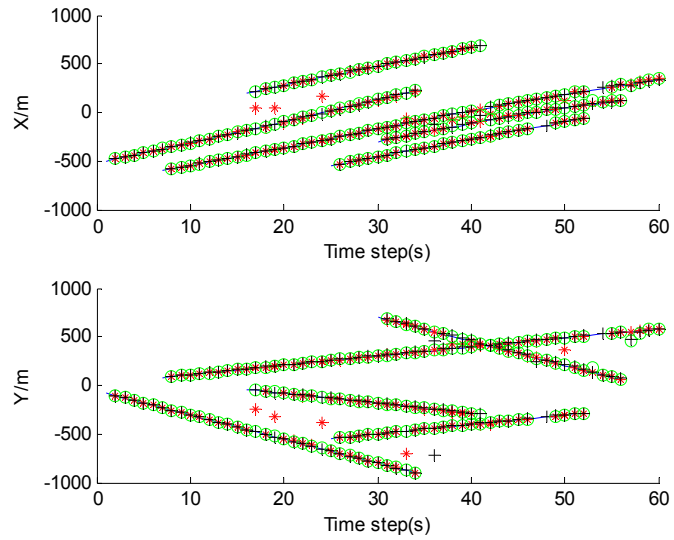

Fig. 2. (b) True targets trajectories and tracking results of SMC-CPHD (+), BP-PHD (*) and BP-CPHD (o) in X and $\mathrm{Y}$ directions.

set to $\mathrm{P}_{\mathrm{d}}=0.99$. The mean estimated target number and mean OSPA distances for different filters are shown in Fig. 3(a) to Fig. 5(b). It can be seen that the OSPA values of the SMC-CPHD filter and the BP-CPHD filter are in general lower than that of the BP-PHD filter with different number of clutter. And the mean OSPA distances increase slowly along with the increase of the number of clutter. The larger the number of the clutter, the worse the performance of the filters is. The OSPA has a little big value when there is a newborn target appearance. The BP-CPHD filter and the SMC-CPHD filter share a similar average OSPA distance. However, the BP-CPHD filter needs much less particle number and runtime than the SMC-CPHD filter as seen in Tab. 3. Both of the box-particle methods use less time and smaller number of particles than the traditional Sequential Monte Carlo method. Figure 3(a) shows that all the three methods are able to get a relative accurate estimate of target number when $r=3$. When the number of the clutter rise to $r=8$ and 12, the BP-CPHD filter and SMC-CPHD filter have an advantage over the BP-PHD filter especially in estimating target number. It can be seen from Fig. 4(a) and Fig. 5(a). Compared to CPHD, PHD is more sensitive to clutter. The cardinalized distribution, rather than the weight of particle, is used to estimate the number of targets in CPHD. It reduces the influence of clutter to estimate the number of targets. This experiment demonstrates that the proposed algorithm is superior to the other two methods in different clutter rates. In addition, the runtime of three filters is increased with the increase in the number of the clutter because more measurements generated by the clutter need to be handled.

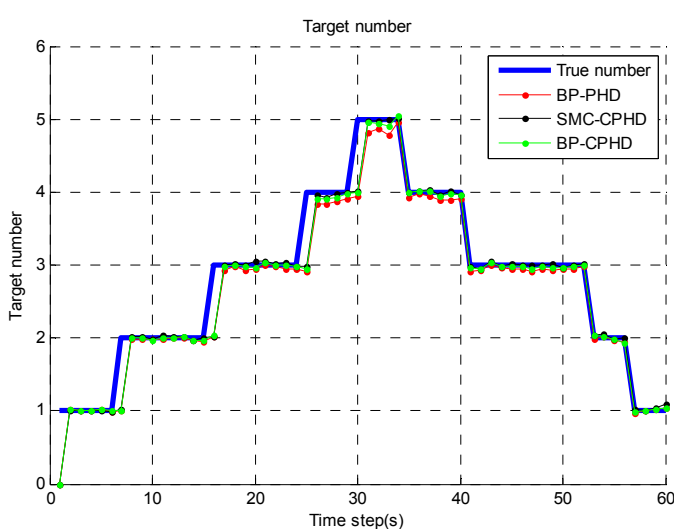

Fig. 3. (a) Mean estimated number of target under $r=3$ and $P_{d}=0.99$.

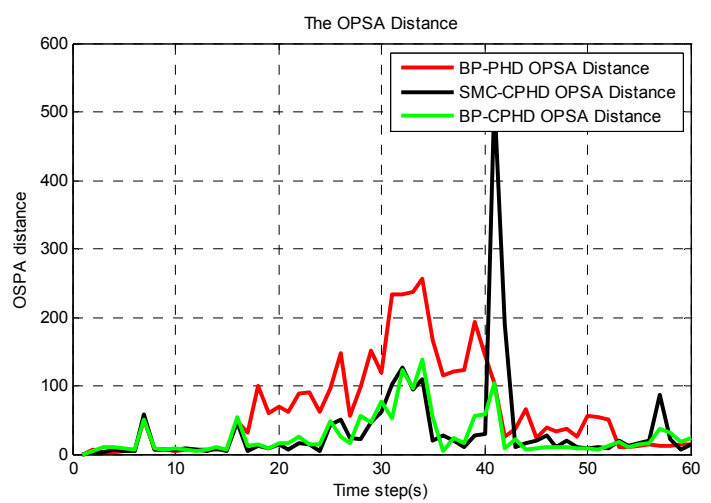

Fig. 3. (b) Mean OSPA distance under $r=3$ and $\mathrm{P}_{\mathrm{d}}=0.99$. 


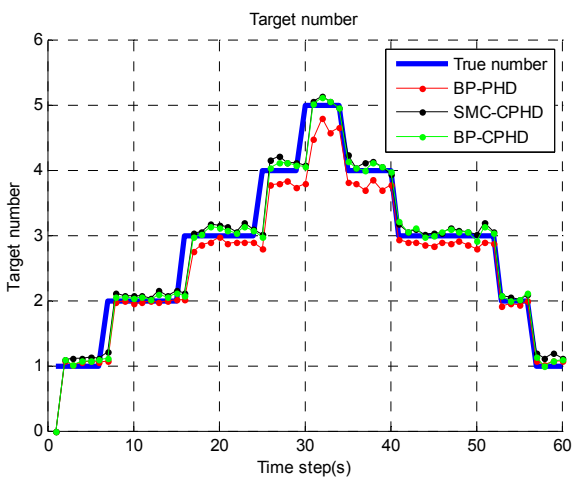

Fig. 4. (a) Mean estimated number of target under $r=8$ and $P_{d}=0.99$.

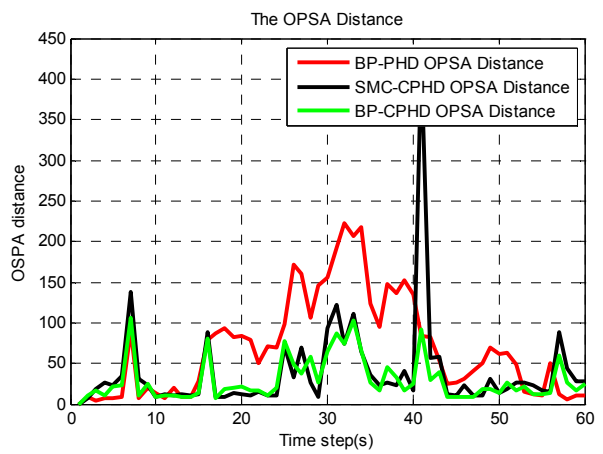

Fig. 4. (b) Mean OSPA distance under $r=8$ and $\mathrm{P}_{\mathrm{d}}=0.99$.

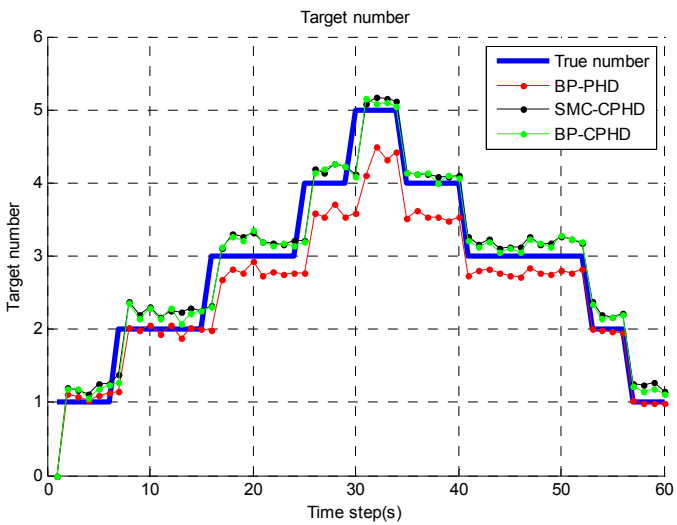

Fig. 5. (a) Mean estimated number of target under $r=12$ and $P_{d}=0.99$.

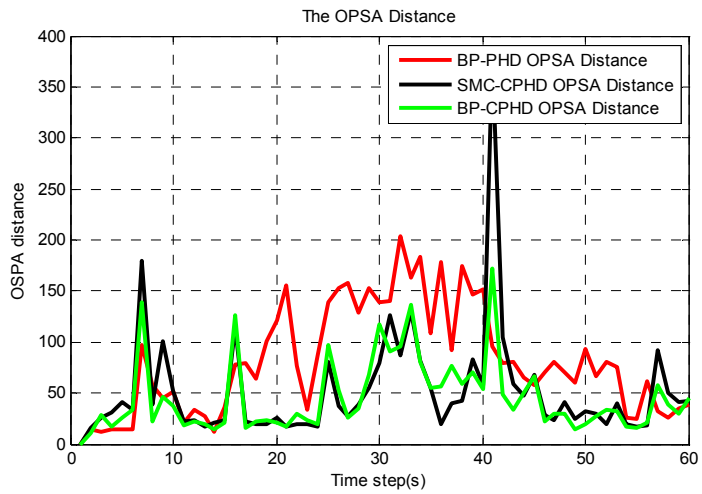

Fig. 5. (b) Mean OSPA distance under $r=12$ and $\mathrm{P}_{\mathrm{d}}=0.99$.
2) Comparing the performance of the three filters with different probability of detection: In this experiment below we investigate the performance of BP-CPHD filter, BP-PHD filter and SMC-CPHD filter with different probability of detection $\mathrm{P}_{\mathrm{d}}=0.95$ and 0.90 , respectively. The number of the clutter is set to $r=3$. Figure 6(a) and (b) show that the estimated number for all the three methods are inaccurate under a low probability of detection. The lower the probability of detection, the worse the estimate of the target number is. It's obvious that the traditional SMC methods outperformed the other two box-particle methods. This is because the number of particles the traditional SMC method used is much larger than that of the box-particle methods. Though the probability of detection is low, there are still a lot of particles to approximate the filter in the traditional SMC methods for its large particle number. However, for Box-particle filter, with the decline of detection probability the box-particle number detected is not enough to approximate the probability density function. Therefore it needs increase of the box-particle number to improve the tracking performance. Of cause the adding box-particle number is relatively small compared to the thousands of particle number. Figure 6 also demonstrates that the performance of the BP-CPHD filter and the SMCCPHD filter is better than that of the BP-PHD filter. These results lead to the conclusion that the proposed BP-CPHD filter is more reliable than BP-PHD filter. Furthermore, taking the runtime into consideration comprehensively, the BP-CPHD filter has a good performance.

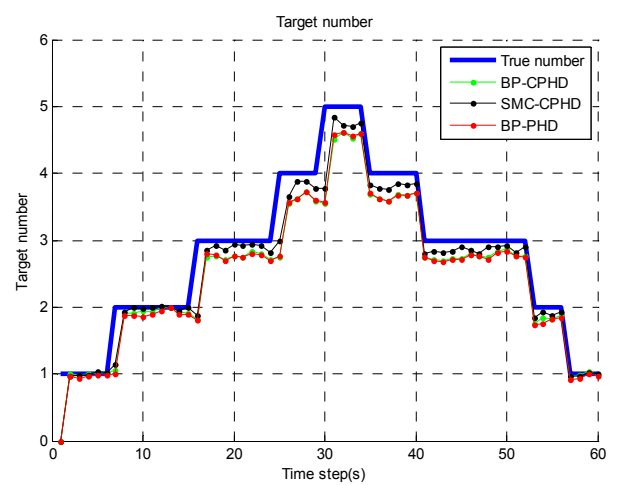

Fig. 6. (a) Mean estimated number of target under $r=3$ and $P_{d}=0.95$.

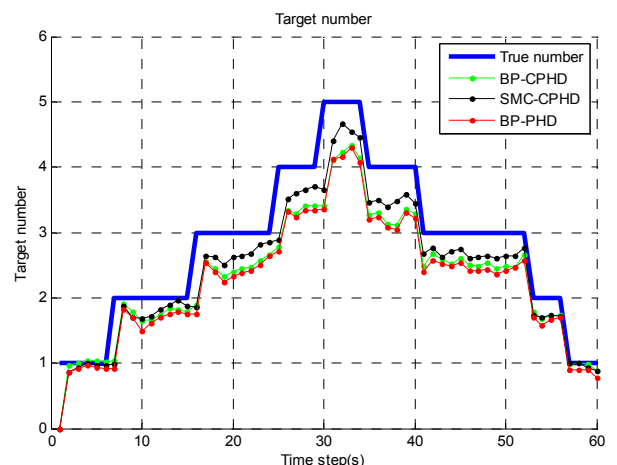

Fig. 6. (b) Mean estimated number of target under $r=3$ and $\mathrm{P}_{\mathrm{d}}=0.90$. 


\section{Conclusions}

This paper presents a novel approach for nonlinear multi-target tracking based on box particles, called boxparticle CPHD (BP-CPHD) filter. It is based on the random finite set theory, and the interval analysis is used to get a box-particle implementation of the CPHD filter. Compared with the SMC-CPHD filter, the number of the particles is decreased greatly. As a result, it is able to guarantee a similar accuracy with less average running time. Experiments demonstrate that the BP-CPHD filter has a higher degree of accuracy and more accurate estimate to the number of targets than the BP-PHD filter, especially in dense clutter environment. Experiments in different probability of detection imply the box-particle based filters need increase of the box-particle number to achieve good tracking performance in low detection probability. In addition, the number of the clutter had an influence on the runtime. So the estimates of the clutter number should be taken into consideration to make the BP-CPHD algorithm agree with actual situation in the future.

\section{Acknowledgments}

The work presented in this paper was supported by the National Science Foundation of China (No. 61372003) and the Fundamental Research Funds for the Central Universities (JB140221). The authors would also like to thank the reviewers who helped to improve and clarify this article.

\section{References}

[1] MAHLER, R. Statistical Multisource-Multitarget Information Fusion. Artech House, 2007.

[2] MAHLER, R. Multi-target Bayes filtering via first-order multitarget moments. IEEE Transactions on Aerospace and Electronic Systems, 2003, vol. 39, no. 4, p. 1152-1178. DOI: 10.1109/TAES.2003.1261119

[3] MAHLER, R. PHD filters of higher order in target number. IEEE Transactions on Aerospace and Electronic Systems, 2007, vol. 43, no. 3, p. 1523-1542. DOI: 10.1109/TAES.2007.4441756

[4] MAHLER, R. A survey of PHD filter and CPHD filter implementations. In Proceedings of SPIE 6567, Signal Processing, Sensor Fusion, and Target Recognition XVI. Florida (USA), 2007, p. 1-12.

[5] VO, B.-N., S. SINGH, S., DOUCET, A. Sequential Monte Carlo methods for multi-target filtering with random finite sets. IEEE Transactions on Aerospace and Electronic Systems, 2005, vol. 41, no. 4, p. 1224-1245. DOI: 10.1109/TAES.2005.1561884

[6] VO, B.-N., MA, W.-K. The Gaussian mixture probability hypothesis density filter. IEEE Transactions on Signal Processing, 2006, vol. 54, no. 11, p. 4091-4104. DOI: 10.1109/TSP.2006.881190

[7] VO, B.-T., VO, B.-N., CANTONI, A. Analytic implementations of the cardinalized probability hypothesis density filter. IEEE
Transactions on Signal Processing. 2007, vol. 55, no. 7, p. 3553-3567. DOI: 10.1109/TSP.2007.894241

[8] GNING, A. RISTIC, B., MIHAYlOVA, L., FAHED, A. An introduction to box particle filtering. IEEE Signal Processing Magazine, 2013, vol. 30, no. 4, p. 166-171. DOI: 10.1109/MSP.2013.2254601

[9] ABDALlAH, F., GNING, A., BONNIFAIT, P. Box particle filtering for nonlinear state estimation using interval analysis. Automatica, 2008, vol. 44, p. 807-815. DOI: 10.1016/j.automatica.2007.07.024

[10] GNING, A., MIHAYlova, L., ABDAllah, F. Mixture of uniform probability density functions for nonlinear state estimation using interval analysis. In Proceedings of the 13th International Conference on Information Fusion. Edinburgh (UK), July 2010.

[11] GNING, A. RISTIC, B., MIHAYLOVA, L. Bernoulli particle / box-particle filters for detection and tracking in the presence of triple measurement uncertainty. Transactions on Signal Processing, 2012, vol. 60, no. 5, p. 2138-2151. DOI: 10.1109/TSP.2012.2184538

[12] SCHIKORA, M., GNING, A., MIHAYLOVA, L., CREMERS, D., $\mathrm{KOCH}, \mathrm{W}$. Box-particle PHD filter for multi-target tracking. In Proceedings of the IEEE 15th International Conference on Information Fusion (FUSION). Singapore, 2012, p. 106-113.

[13] LI-PING SONG, XUE-GANG ZHAO. Box-particle cardinality balanced multi-target multi-Bernoulli filter. Radioengineering, 2014, vol. 23, no. 2, p. 609-617.

[14] MOORE, R. E., CLOUD, M. J., KEARFOTT, R. B. Introduction to Interval Analysis. Siam, 2009. ISBN-10: 0898716691

[15] VO, B.-N., MA, W.-K. The Gaussian mixture probability hypothesis density filter. IEEE Transactions on Signal Processing, 2006, vol. 54, no. 11, p. 4091-4104. DOI: 10.1109/TSP.2006.881190

[16] JAULIN, L., KIEFFER, M., DIDRIT, O., WALTER, E. Applied Interval Analysis: With Examples in Parameter and State Estimation, Robust Control and Robotics. London: Springer, 2001. DOI: 10.1007/978-1-4471-0249-6

[17] GNING, A. RISTIC, B., MIHAYLOVA, L. A box particle filter for stochastic set-theoretic measurements with association uncertainty. In Proceedings of the 14th International Conference on Information Fusion. Chicago (IL, USA), July 2011, 8 p. Print ISBN: 978-1-4577-0267-9

[18] SCHIKORA, M., GNING, A., MIHAYLOVA, L., CREMERS, D., $\mathrm{KOCH}, \mathrm{W}$. Box-particle probability hypothesis density filtering. IEEE Transactions on Aerospace and Electronic Systems, 2014, vol. 50, no. 3, p. 1660-1672. DOI: 10.1109/TAES.2014.120238

[19] PETROV, N., MIHAYlOVA, L., DE FREITAS, A., GNING, A., Crowd tracking with box particle filtering. In Proceedings of the 17th International Conference on Information Fusion (FUSION). Salamanca (Spain), 2014, 7 p.

[20] PETROV, N., MIHAYlOVA, L., GNING, A. Rectangular extended object tracking with box particle filter using dynamic constraints. IET Conference Publication 629:IET Conference on Data Fusion and Target Tracking (DF\&TT 2014). Liverpool (UK), 2014, 7 p. ISBN: 978-1-63266-664-2.

[21] MAHLER, R. General Bayes filtering of quantized measurements. In Proceedings of the 14th IEEE International Conference on Information Fusion (FUSION). Chicago (IL, USA), July 2011, 7 p. Print ISBN: 978-1-4577-0267-9

[22] MENG LIANG, LIPING SONG, HONGBING JI, YUFEI WANG. Box-particle CPHD filter for multi-target tracking, In Proceedings of 2015 International Conference on Control, Automation and Information Sciences (ICCAIS). Changshu (China), 2015, p. 80-84. DOI: 10.1109/ICCAIS.2015.7338730 
[23] PALlaUf, J., WAGNER, J., LEON, F.P. Evaluation of statedependent pedestrian tracking based on finite sets. IEEE Transactions on Instrumentation and Measurement, 2015, vol. 64, no. 5, p. 1276-1284. DOI: 10.1109/TIM.2014.2375712

[24] SUN, J.G., LIU, J., ZHAO, L.Y. Clustering algorithms research. Journal of Software, 2008, vol. 19, no. 1, p. 48-61. DOI: 10.3724/SP.J.1001.2008.00048

\section{About the Authors ...}

Li-ping SONG was born in 1975 in Xi'an, China. He received his M.Sc. degree in Signal Processing from Xidian University in 2003. He received the Ph.D. degree in Pattern Recognition and Intelligent Systems from Xidian University in 2008 . He is currently an associate professor of Xidian University. His research interests include signal processing, target tracking and nonlinear filtering.

Meng LIANG was born in 1991. She is a Master Degree Candidate at the School of Electronic Engineering at Xidian University. Her research interests include particle filter and target tracking.

Hong-bing JI was born in 1963. He received his M.Sc. degree in Signal Processing from Xidian University in 1989. He received the Ph.D. degree in Signal and Information Processing from Xidian University in 1999. He is currently a professor of Xidian University. His research interests include passive multiple sensor detection and target tracking, intelligent information processing and pattern recognition. 